\title{
Research on Emergency Resource Scheduling in Smart City based on HPSO Algorithm
}

\author{
Hong Ye \\ School of Information Science and Technology, Dalian Maritime University \\ Dalian, Liaoning, China, 116026 \\ yehong@dlmu.edu.cn
}

\begin{abstract}
Smart city as a novel further urban development pattern paid more attention by governments, is no longer confined to the virtual concept frameworks, and is now realized and proved effectively in different domain, especially in emergency management. Based on current research of emergency management in smart city system, with considering the new features of emergency resources, such as real time, distributed, self-organization, etc., a emergency management system architecture is proposed firstly, which composes of resource layer, sense and network layer, smart service layer, application layer and social management layer. Then a clear collaborative emergency resource scheduling model is constructed to minimize the time, cost and maximize the quality of resource. Moreover, we present an improved HPSO to solve the multi-objective emergency resource scheduling problem. Finally, a simulation experiment is carried out to obtain the evaluation results which can indicate us that our scheduling model and algorithm are applicative and valuable in emergency management of smart city.
\end{abstract}

Keywords: WSNs, non-equilibrium statistical mechanics method, performance evaluation

\section{Introduction}

In recent years, with the rapid development of network information technology, such as IoT, cloud computing, big data and so on, city presents a development trend of networking, distributed, intelligence [1]. And the new concept and development model called smart city (SC) has been proposed and become the subject of increasing attention and it now appears as a new paradigm of intelligent city development and sustainable socio-economic growth $[2,3]$. Nowadays, the large and small districts are proposing the new development frameworks and concept model, called smart city, which represents a community of average technology size, interconnected and sustainable, comfortable, attractive and secure. For example, the United States, Britain, Japan and Korea put emphasis on the next generation of internet, and speed up the smart city systems, which is to strength city management, improve city service and ameliorate city function, especially in smart grid, smart education, smart transportation and so on [4]. And there are only a few research on smart city mainly concentrated in the concept, key technology, application prospects, which seriously hindered the further development of the city $[5,6]$.

As we know, a smart city should be able to optimize the use and exploitation of both tangible (e.g., transport infrastructures, energy distribution networks, natural resources) and intangible assets (e.g., human capital, intellectual capital of companies, and organizational capital in public administration bodies) in real time anywhere. It opens up a wealth of 
opportunities for different emergency management applications, such as flood, fire, earthquake emergency rescue and disaster relief, anti-terrorism, remote control of hazardous area and so on [7]. More attention has been paid to the emergency resource scheduling and allocation, and the smart city is no longer confined to the traditional area, which can predict the coming emergency event and real time management by using the new information technology and smart city system that cannot be realized in the past. Emergency resource is the supporting element after the accident, the efficiency of its allocation and scheduling is one of the primary embodiments of emergency response capacity $[8,9]$. Besides the object of emergency management is to get the whole success because the derivative emergency often occurs and many kings of danger are interweaved. The time that resource reaches the demand and the amount are the most important factors related to the efficiency of emergency management [10, 11]. Lots of research works have been done in the field, Zhang and Guangliang researched the configuration and scheduling of emergency resources under a fire disaster by establishing and analyzing a dynamic model $[12,13]$. Shan, et al., built emergency resource scheduling models under the constraints of the emergency rescue starting time and the rescue team number, respectively $[14,15]$. Rachaniotis et al. presented a solution for emergency resource allocation among multiple disaster places to solve the conflict in allocation [16]. Emergency resource scheduling supported in distributed smart city environment is still remained as an open field of research from diverse perspectives.

In summary, current works of emergency resource allocation is in the beginning stage, there is considerable problem space to explore and solve. Most of these studies focused on the expansion of characteristics of emergency resources based on traditional models and the algorithm designing for task dispatch in centered environment $[17,18]$. However, in smart city, emergency tasks and resources have new features such as dynamic, distributed, real time and so on. Therefore, we should firstly construct a new emergency management system model in smart city to better schedule service tasks and allocate emergency resources in real time and dynamic manner. Secondly, a more comprehensive emergency resource scheduling model based on three objective functions is proposed to satisfy different users' request, and then the HPSO method is used to search the optimal resource composition in the candidate resource set. Finally, we implement some emergency management simulation experiments to prove that the proposed model and improved HPSO are efficient and effective, which provides an excellent theory foundation and practical experience.

\section{Emergency Management System Architecture in Smart City}

In a smart city system, various city resources and capabilities can be intelligently sensed and connected into the wider internet, and automatically managed and controlled by using IoT and could computing technology. Then the emergency resources can be virtualized and encapsulated into emergency cloud resources pool that can be accessed, invoked, deployed, and on-demand used [19]. The emergency cloud resources are classified and aggregated according to specific rules and algorithms. Different users can search and invoke the qualified resource from a related emergency cloud according to their requirements, and assemble them to be a virtual smart city environment in the whole lifecycle of emergency events [20,21].

With the new point mentioned above in mind, we present novel five-layered emergency management system architecture to better understand and analyze the emergency tasks and resource scheduling in smart city, and describe also the essential key technologies used in which includes emergency resource layer, sense and network layer, emergency 
service layer, application system layer and user layer. Figure 1 shows us the key elements of the system framework:

1) Resource layer: This component is responsible for collection of related emergency resource provided by a variety of suppliers distributed in different regions such hospital, government, police and so on. Using IoT, cloud computing and virtualize technology, emergency resource can be further bonded as cloud resource in emergency system platform.

2) Sense and network layer: this component first sense and identify the emergency resource distributed in smart city and analyze the resource state using related method embed in the system. Then, massive information need to be processed and delivered to the upper layer, namely emergency service layer through the local and external network.

3) Emergency service layer: this component includes resource discovery, resource composition, trust management, resource allocation, resource binding, reputation mechanism and safe control. It is the core element of the system and support the scheduling model.

4) Application system layer: This component is responsible for dynamic allocation and management of emergency resource, real control and auto-recovery, performance monitoring evaluation and emergency collaboration which can deal with emergency events effectively.

5) User layer: this layer includes smart city interaction network, city event system and emergency management system, in which we can interact with others, obtain the new information of emergency events occurring right now and submit a service request; meanwhile, government can schedule emergency resource to different task in different area.

Scheduling mechanisms should know the status of each element/resource in the distributed smart city environment and, based on them, intelligently apply algorithms to better allocate emergency resources to tasks according to their pre-established requirements [22]. This way, we may consider that emergency resources, resource modelling, application requirements, and provider requirements constitute the input used by a resource allocation mechanism. These resources are located in a distributed pool and shared by multiple users, and each provider is free to model its resources according to its business model.

\section{Collaborative Emergency Resource Scheduling Model}

In this paper, the distributed emergency resource scheduling in smart city is deemed to be highly heterogeneous and with resources of uncertain information. The scheduling objectives are multiple; specifically the focus is on two objectives: minimizing the complete time, cost and resource consumption. The task is to search the optimal set of this MOO problem under the considered emergency environment constraints in smart city [23].

\subsection{Problem Formulation}

Collaborative emergency resource scheduling is determining how to distribute the processing emergency tasks to resource nodes or providers, who belong to diverse emergency resource alliance [23]. With the help of the task planner, emergency tasks will be reasonable decomposition. Then, we match and find suitable resources to optimal select a set of effective solution in many candidate providers. The resource scheduling center is considered to have two pieces of information: a collection of user requests and processor information. Each user request is represented by a directed acyclic graph (DAG), which captures a number of task units involved, each unit's own properties, and the relationships among task units. One essential property of each emergency task unit that we must take into account for assignment is the task type. Figure 2 is a collaborative emergency task allocation example, when the system platform receives task requests $T R$, the emergency task planner decompose $T R$ into $n$ sub emergency tasks. Each sub emergency task can be finished by lots 
of candidate emergency resources. In Figure 1, an example of DAGs, each circular node represents a task unit, which has its task type; each rectangular node represents an emergency resource or service, each directed line between two emergency nodes represents their dependency relationship, and we can add weight to the edges to depict the flow size.

$V=\left(V_{i} \mid 1=1: n\right)$ represents the decomposed emergency task units of each user request, where $n$ is the total number of task units. $T=\left(T_{1} \mid 1=1: n\right)$ denotes the task type of each unit in $V$, where $T_{1} \in\left(1,2, \cdots, T_{\max }\right)$ with $T_{\max }$ indicating the total number of task types. $E(n \times n)$ denotes dependencies between emergency task units in $V$. Let $E_{i j}=1$, if data obtained from $V$ is used by $V_{j}$. Otherwise, $E_{i j}=0 . D I N(n \times n)$ represents each task unit's input data size. $\operatorname{DOUT}(n \times n)$ represents each task unit's output data size. $P=\left(P_{i} \mid 1=1: p\right)$ represents a collection of emergency resource nodes, where $p$ is the total number of resource nodes. $T P\left(T_{\max } \times p\right)$ denotes the actual capacity of the node, where $T P_{i k}$ represents time cost for resource node $P_{k}$ to execute the task unit of type $i . T P_{k}$ represents the average capacity of resource node $k$, whose value can be obtained by calculating the mean of elements in column $k$ of matrix TP.S denotes the resource size of each node. $E P\left(T_{\max } \times p\right)$ denotes the emergency resource consumption rate, where $E P_{i k}$ represents the resource consumed on node $P_{k}$ by completing task unit of type $i$ per unit time per unit data. $D C$ indicates the channel between nodes, where $D C_{k l}$ represents the transferring rate of resource from node $P_{k}$ to node $P_{l} . E C$ denotes the communication energy consumption rate, where $E C_{k l}$ represents the energy consumed by transferring information from node $P_{k}$ to node $P_{l}$ per unit time per unit information. $X$ describes the mapping between task units and resource nodes. $X(i)=K$ means that task unit $V_{i}$ has been assigned to node $P_{k}$ to be executed.

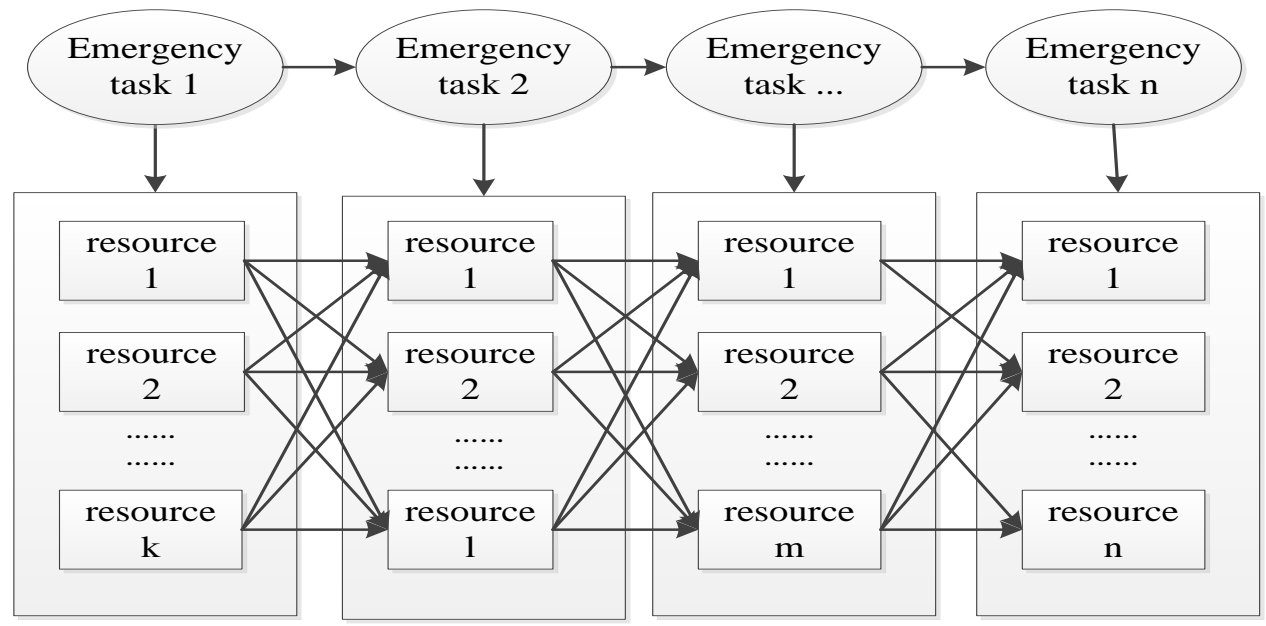

Figure 2. Collaboration Emergency Task Scheduling Process

\subsection{Objective Functions and Optimization Model}

Collaborative emergency resource and task allocation has two principles: First, the optional emergency resource allocation plan must achieve the shortest time, the lowest cost and highest quality in many service suppliers; second, to solve the problems in the emergency process, we should make rapid redistribution when emergency situation changes, and 
finish an emergency task in time. According to the two principles, this paper establishes an optimization model, taking $\mathrm{T}$ (emergency task completion time), Q (quality of emergency resource and task), $\mathrm{C}$ (total resource cost) as objective function.

1) The shortest total time $T$

$$
\min T=\sum_{i=1}^{n} \sum_{j=1}^{m_{i}} x_{i j} t_{i j}
$$

In which $x_{i j} \in(0,1)$, denotes that for sub task $i$, if we choose the $j$ node to complete, then $x_{i j}=1$, otherwise $x_{i j}=0 . t_{i j}$ represents complete time to the $j$ node, $n$ is the number of all sub tasks.

2) The lowest cost $C$

$$
\min C=\sum_{i=1}^{n} \sum_{j=1}^{m_{i}} x_{i j} c_{i j}
$$

In which $c_{i j}$ represents complete cost for the $j$ node to finish the task.

3) The highest quality $Q$

$$
\operatorname{maxQ}=\sum_{i=1}^{n} \sum_{j=1}^{m_{i}} x_{i j} q_{i j}
$$

In which $q_{i j}$ represents complete quality for the $j$ node to finish the task. In the multiobjective optimization process, because each target has different dimension, we need unify objective function index, and transfer multi-objective problem into a single objective by weighted algorithm. In order to make a unified objective function, using the following normalization method:

$$
\begin{gathered}
b_{i j}=\left(r_{\max }-r_{i j}\right) /\left(r_{\max }-r_{\min }\right) \\
b_{i j}=\left(r_{i j}-r_{\min }\right) /\left(r_{\max }-r_{\min }\right)
\end{gathered}
$$

Finally, the whole objective function is as following:

$$
\operatorname{minf}(x)=w_{t} T+w_{c} C+w_{q} Q
$$

In which $w_{t}, w_{c}, w_{q}$ is respectively weight of three targets, and $w_{t}+w_{c}+w_{q}=1$.

\section{Hybrid Approach}

As presented previously, our goal is to select available resource from each $P$ by minimizing the objective function $f(x)$ on the premise of satisfying the multi-constraint. So, the main idea of our approach is adopting efficient search algorithm to seek the optimal solution among the feasible resource combinations. Motivated by the above description, the whole selection framework mainly consists of two steps. First, we construct the fixed tabu list according to relational constraint rules, the fixed tabu list is used to record the infeasible combinations. Then, we utilize a hybrid particle swarm optimization algorithm to search a solution.

\subsection{Construct the Fixed Tabu List}

In order to search an optimal solution among the feasible combinations, we create a fixed tabu list to record the combinations which against the relational constraint rules in $P$. The fixed tabu list includes several tabu tuples and a tree index. A tabu tuple represents a set of infeasible combinations, and the tree index can improve the speed of tuple retrieval 
operations on the fixed tabu list. During the construction, the tabu tuples can be converted from dependent rule or conflict rule with following formulas.

$$
\left\{\begin{array}{l}
\forall C S \exists s \in C S(\operatorname{drs}(s) \cap C S \neq d r s(s)) \rightarrow \text { Infeasible }(C S) \\
\forall C S \exists s \in C S(\operatorname{crs}(s) \cap C S \neq \phi) \rightarrow \text { Infeasible }(C S)
\end{array}\right.
$$

Where $C S$ represents any composite resource in $P$, Infeasible (CS) means the composite resource $C S$ is infeasible, so it cannot be a potential solution. Base on the above formula, we adapt the tactic of divide and rule to create the tabu tuples with the different types of rules.

After the tuple creation process, we build a tree index for these tuples according to the conflict services and dependent services, which belong to the same services class. The tree index can improve the performance of lookup speed that means, whether the composite service is a feasible combination can be quickly checked through the tree index

\subsection{Searching the Optimal Solution}

After the above procedures, we propose a Hybrid Particle Swarm Optimization Algorithm (HPSO for short) to solve this scheduling problem with the characteristics presented in this paper. This algorithm is an extended version of PSO, which not only employs a mutation mechanism to improve population's the quality and the diversity, but also employs a time decreasing strategy for setting the parameters.

\section{1) Problem Representation}

One of the key issues in using HPSO algorithm is the representation of the problem. To let the HPSO search for a solution, we first need to encode the problem with a suitable particles. In our case, the particle represented by an integer array with a number of items equals to the number of service class. Each item, in turn, contains an index to the array of the candidate resources in distinct emergency resource class.

\section{2) Particle Movement}

As a fitness function, $f(x)$ can use to guide movement of particles in the iterative process. During the iterative process, each particle keeps the track of its permutation and fitness value in the problem space which are associated with the best fitness value it has achieved so far. That is called pbest of this particle. Besides another best value is call gbest is also tracked by the particle swarm optimizer, which is the best value, obtained so far by any particle in all the population of the particles. Base on the best values, the velocity and position of any particle $X$ are updated with following equations where $V^{t+1}$ is the new velocity of the current particle, $X^{t+1}$ is the new position of particle $X$.

$$
\begin{aligned}
& V^{t+1}=\left(\omega \otimes V^{t}\right) \circ\left(c_{1} \otimes\left(X_{\text {pbest }} \Theta X^{t}\right)\right) \circ\left(c_{2} \otimes\left(X_{\text {gbest }} \Theta X^{t}\right)\right) \\
& X^{t+1}=X^{t} \oplus V^{t+1}
\end{aligned}
$$

Generally, more exploration is needed in the initial stages when the algorithm has very little knowledge about the search space. In contrast, more exploitation is needed during the later stages so that the algorithm is able to exploit the information it has gained so far, and make better global exploration of the search space. Hence, we employ a time decreasing inertia weight value calculated as follows:

$$
\omega=\omega_{\max }-\left(\omega_{\max }-\omega_{\min }\right) \frac{t}{G}
$$


Where $t$ is the generation index representing the current number of evolutionary generations, and $G$ is a predefined maximum number of generations. Here, the maximal and minimal weights $\omega_{\max }$ and $\omega_{\min }$ are usually set to 0.85 and 0.25 .

3) Optimized the Population

Since a particle may not be a feasible solution, and the gbest always have the same service permutations with its pbest that make the direction of the gbest's movement blind. The mutation operation is used to optimize the particle in the population. And it can keep the position of the particles not in the fixed tabu list, and enrich the diversity of the population.

\section{4) HP SO for Multi-constraint Web Service Selection}

The procedure of HP SO for the multi-constraint Web service selection is described in Figure 3. After the problem representation, every particle in the swarm is randomly assigned in the initialization phase of the algorithm. Then, the particles in the population are optimized by mutation operation, and their fitness is calculated and evaluated by $f(x)$. The local and global best positions are set and the time is initialized to 1 . For every particle a new velocity is calculated with equation (9).The particle is moved according to this new velocity. After moving the entire swarm, the time is incremented by 1 , and the optimized the population is working. As long as no solution is found or a predefined stopping criterion is not met, this procedure is repeated.

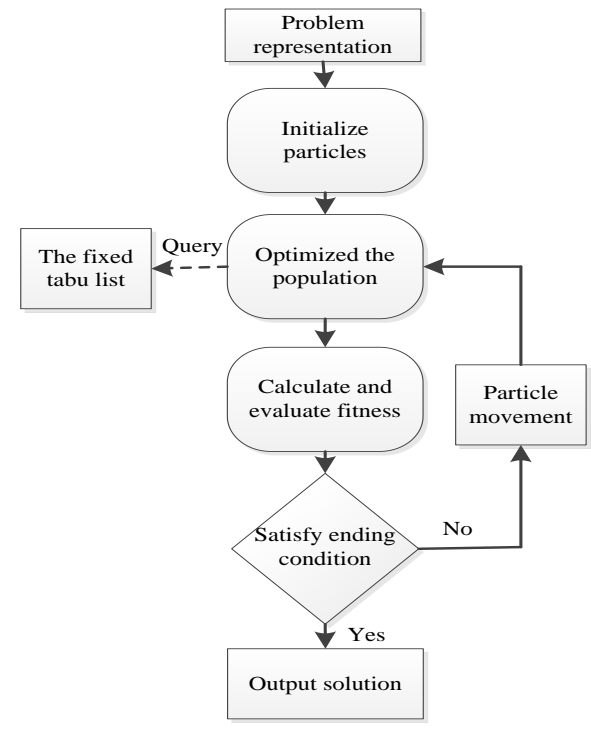

Figure 3. HPSO for Multi-constraint Emergency Resource Scheduling

\section{A Numerical Example Analysis}

In order to obtain a large number of accurate real-time data, in this paper, we construct an emergency management simulation in smart city environment by using the network simulator. For the purpose of our evaluation, we consider a scenario that is a composite application comprises emergency resources from 7 different resource classes with a sequence structure. And we compare the efficiency of the following selection methods: RGA that is a standard GA optimization method, and HGA that is a hybrid genetic algorithm. The simulation is performed in the laboratory including common software and hardware 
environment, namely CPU Intel core $4.0 \mathrm{GHz}$, and memory for the DDRII4G, operating system is Windows7.0 professional edition, using the MATLAB command.

\subsection{Sample Data}

In order to better explain the actual situation, we assume that the emergency resource management system gets 7 emergency task requests; it means that there are 7 tasks to meet, and there are 15 kinds of resource to meet all tasks, in which there 6 kinds of shared resource. The complete time, cost, quality of each emergency resource can be randomly generated by using simulation platform, and then we can collect related data in Table 1.

Table 1. Simulation Data of Emergency Resources

\begin{tabular}{|c|c|c|c|c|}
\hline Emergency task number & Emergency resource name & Time & Cost & Quality \\
\hline \multirow{2}{*}{1} & $\mathrm{R}_{1} \quad$ (shared resource) & 36 & 40 & 6 \\
\hline & $\mathrm{R}_{2}$ & 63 & 32 & 6 \\
\hline \multirow{2}{*}{2} & $\mathrm{R}_{3}$ & $\ldots$ & $\ldots$ & $\ldots$ \\
\hline & $\mathrm{R}_{4}$ (shared resource) & 65 & 24 & 5 \\
\hline \multirow{2}{*}{3} & $\mathrm{R}_{5}$ & 55 & 26 & 4 \\
\hline & $\mathrm{R}_{6}$ (shared resource) & 33 & 54 & 6 \\
\hline \multirow{2}{*}{4} & $\mathrm{R}_{7}$ & $\ldots$ & $\ldots$ & $\ldots$ \\
\hline & $\mathrm{R}_{8}$ (shared resource) & 56 & 42 & 6 \\
\hline \multirow{3}{*}{5} & $\mathrm{R}_{9}$ & 33 & 74 & 8 \\
\hline & $\mathrm{R}_{10}$ (shared resource) & 36 & 25 & 5 \\
\hline & $\mathrm{R}_{11}$ & $\ldots$ & $\ldots$ & $\ldots$ \\
\hline \multirow{2}{*}{6} & $\mathrm{R}_{12}$ (shared resource) & 51 & 42 & 6 \\
\hline & $\mathrm{R}_{13}$ & 43 & 34 & 3 \\
\hline \multirow{2}{*}{7} & $\mathrm{R}_{14}$ & 74 & 16 & 7 \\
\hline & $\mathrm{R}_{15}$ & 24 & 84 & 9 \\
\hline
\end{tabular}

\subsection{Comprehensive Evaluation and Analysis of the Results}

In Figure 3, we can see clearly that the run time of the three algorithms all are increasing with resource nodes. However, the time of other two algorithms grows exponentially; meanwhile our algorithm is stable, and the run time grows smooth and has smaller fluctuations, which certify our model and algorithm is better and efficient.

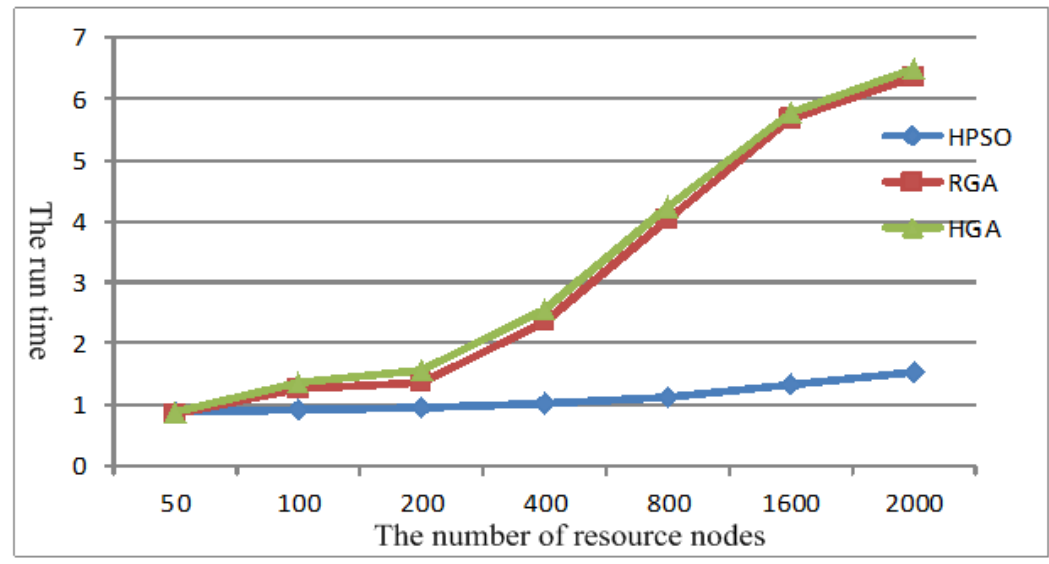

Figure 4. Time Curve with Increase of Resource Nodes 
As shown in Figure 5, our algorithm tends to be stable after 100 iterations; however, RGA and ant HGA are iterated 150 times and 200 times. In addition, the average time to search the global optimal solution by the RGA is $3.854 \mathrm{~s}$, the probability of optimal solution is 0.46 ; the average time to search the global optimal solution by HGA is $4.587 \mathrm{~s}$, the probability of optimal solution is 0.53 . However, the average time to search the global optimal solution by our HPSO is $1.526 \mathrm{~s}$, the probability of optimal solution is 0.72 . This method is better than the other two algorithms, and we get the best emergency resource allocation plan is $\left(R_{13}, R_{25}, R_{33}\right.$, $R_{46}, R_{51}, R_{68}, R_{71}$ ), which provides a perfect decision for the emergency manager in smart city.

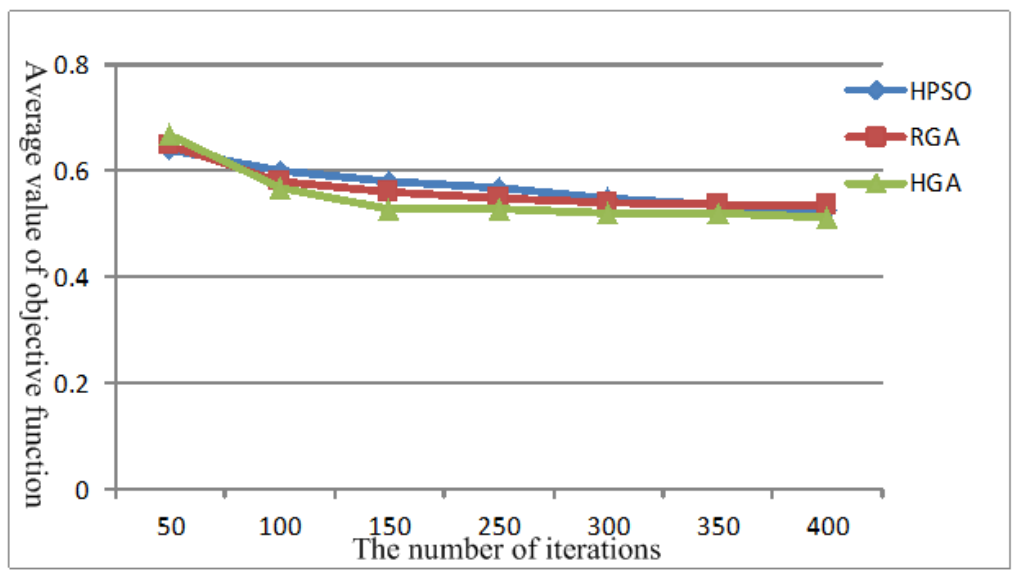

Figure 5. Algorithm Performance Curve

\section{Conclusion}

As a new paradigm of intelligent urban development and sustainable socio-economic growth, smart city is changing our life in different domains, especially in emergency. Emergency management is presenting new characteristics in smart city, such as real time aware, distributed, self-organization, leading to difficult problem in emergency tasks and resources scheduling under new situation, which is deemed to be highly heterogeneous and with resource of uncertain state information. Based on current research of emergency resource scheduling in center city system, in this paper, emergency management system architecture is first constructed to adopt the different context, which provides another research point. According to the actual environment, we build collaborative emergency resource scheduling model considering the task relations. Then, an improved HPSO is applied to solve the multi-objective NP-hard problem. Some simulation experiments are carried out to show us the effect and efficiency of proposed model and improved HPSO in smart city. In this paper it is assumed that in the course of emergency response process, the task remains unchangeable, therefore a possible future direction is to extend the mapping process to handle with dynamic structure of the emergency task. Meanwhile, a new effective algorithm should be put forward in the future to better solve the complex problem.

\section{Acknowledgements}

This work is supported in part by National Natural Science Foundation of China under Grant No.61173034, the Scientific Research Fund of Liaoning Provincial Education Department under Grant No.L2013195, and the Fundamental Research Funds for the Central Universities under Grant No.3132013040. 


\section{References}

[1] P. Vlacheas, R. Giaffreda, V. Stavroulaki, et al., "Enabling smart cities through a cognitive management framework for the internet of things", Communications Magazine, IEEE, vol. 51, no. 6, (2013).

[2] J. M. Shapiro, "Smart cities: quality of life, productivity, and the growth effects of human capital", The review of economics and statistics, vol. 88, no. 2, (2006), pp. 324-335.

[3] A. Luberg, T. Tammet and P. Järv, "Smart City: A Rule-based Tourist Recommendation System", Springer Vienna, (2011).

[4] K. Su, J. Li and H. Fu, "Smart city and the applications", Electronics, Communications and Control (ICECC), 2011 International Conference on. IEEE, (2011), pp. 1028-1031.

[5] A. Resmini and L. Rosati, "Smart City-Cities as Ecosystems and Palimpsests", (2012).

[6] Y. Li and A. Liu, "Analysis of the Challenges and Solutions of Building a Smart City", Bridges, (2014), 10: 9780784413135.149.

[7] T. Nam and T. A. Pardo, "Smart city as urban innovation: Focusing on management, policy, and context", Proceedings of the 5th International Conference on Theory and Practice of Electronic Governance, ACM, (2011), pp. 185-194.

[8] N. Komninos, H. Schaffers and M. Pallot, "Developing a Policy road map for Smart Cities and the future internet", e-Challenges e-2011 Conference Proceedings, IIMC International Information Management Corporation, IMC International Information Management Corporation, (2011).

[9] "Emergency Management: The American Experience 1900-2010", CRC Press, (2012).

[10] J. H. Hu, G. P. Zhong and G. C. Yan, "Design and implementation of dynamic emergency resource scheduling based on GIS", Application Research of Computers, vol. 1, (2012), pp. 057.

[11] L. Zhang, Y. Lin, G. Yang, et al., "Emergency resources scheduling based on adaptively mutate genetic algorithm", Computers in human behavior, vol. 27, no. 5, (2011), pp. 1493-1498.

[12] Z. Guangliang and S. Lian, "Research on Scheduling Models of Emergency Resource", Intelligent Computation Technology and Automation (ICICTA), 2011 International Conference on. IEEE, vol. 1, (2011), pp. 1110-1113.

[13] S. Shan, L. Wang and L. Li, "Modeling of emergency response decision-making process using stochastic Petri net: an e-service perspective", Information Technology and Management, vol. 13, no. 4, (2012), pp. 363-376.

[14] A. M. Caunhye, X. Nie and S. Pokharel, "Optimization models in emergency logistics: A literature review", Socio-Economic Planning Sciences, vol. 46, no. 1, (2012), pp. 4-13.

[15] N. P. Rachaniotis, T. K. Dasaklis and C. P. Pappis, "A deterministic resource scheduling model in epidemic control: A case study”, European Journal of Operational Research, vol. 216, no. 1, (2012), pp. 225-231.

[16] Z. O. Toups, A. Kerne, W. A. Hamilton, et al., "Zero-fidelity simulation of fire emergency response: improving team coordination learning", Proceedings of the SIGCHI Conference on Human Factors in Computing Systems. ACM, (2011), pp. 1959-1968.

[17] I. Adan, J. Bekkers, N. Dellaert, et al., "Improving operational effectiveness of tactical master plans for emergency and elective patients under stochastic demand and capacitated resources", European Journal of Operational Research, vol. 213, no. 1, (2011), pp. 290-308.

[18] L. Sánchez, V. Gutiérrez, J. A. Galache, et al., "SmartSantander: Experimentation and service provision in the smart city", Wireless Personal Multimedia Communications (WPMC), 2013 16th International Symposium on. IEEE, (2013), pp. 1-6.

[19] Y. Wang and Y. Zhou, "Cloud architecture based on Near Field Communication in the smart city", Computer Science \& Education (ICCSE), 2012 7th International Conference on. IEEE, (2012), pp. 231-234.

[20] D. Fuselli, F. De Angelis, M. Boaro, et al., "Action dependent heuristic dynamic programming for home energy resource scheduling”, International Journal of Electrical Power \& Energy Systems, vol. 48, (2013), pp. 148-160.

[21] D. Li, Y. Yao, Z. Shao, et al., "From digital earth to smart earth", Chinese Science Bulletin, vol. 59, no. 8, (2014), pp. 722-733.

[22] M. Brenna, M. C. Falvo, F. Foiadelli, et al., "Challenges in energy systems for the smart-cities of the future", Energy Conference and Exhibition (ENERGYCON), 2012 IEEE International. IEEE, (2012), pp. 755-762. 


\section{Author}

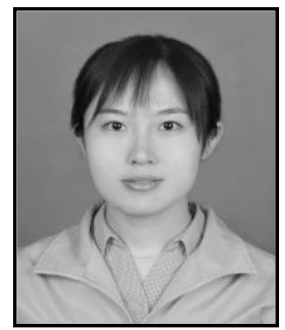

Hong Ye, She received her master's degrees from Dalian University of Technology, China in 2003. Now she is full lecturer school of Information Technology, Dalian Maritime University, China. Since 2009, she has been pursuing the Ph.D. Degree at the Department of Computer Sciences, Dalian Maritime University. Her current research interests include different aspects of Distributed Systems. 
International Journal of Smart Home

Vol. 9, No. 3 (2015) 